\title{
Sufficient conditions for stabilization of sampled-data nonlinear systems via discrete-time approximations
}

\author{
D. Nešić, \\ Department of Electrical and Electronic Engineering, \\ The University of Melbourne, Parkville, 3052, Victoria, Australia \\ A. R. Teel ${ }^{\dagger}$ and P. V. Kokotović $\ddagger$ \\ CCEC, Electrical and Computer Engineering Department, \\ University of California, Santa Barbara, CA, 93106-9560
}

October 3, 2000

\begin{abstract}
Given a parameterized (by sampling period $T$ ) family of approximate discrete-time models of a sampled nonlinear plant and given a family of controllers stabilizing the family of plant models for all $T$ sufficiently small, we present conditions which guarantee that the same family of controllers semi-globally-practically stabilizes the exact discrete-time model of the plant for sufficiently small sampling periods. When the family of controls is locally bounded, uniformly in the sampling period, the inter-sample behavior can also be uniformly bounded so that the (time-varying) sampled-data model of the plant is uniformly semi-globally-practically stabilized. The result justifies controller design for sampled-data nonlinear systems based on the approximate discrete-time model of the system when sampling is sufficiently fast and the conditions we propose are satisfied. Our analysis is applicable to a wide range of time-discretization schemes and general plant models.
\end{abstract}

Keywords: Discrete-time, nonlinear, sampled-data, stabilization.

\section{Introduction}

The three main approaches for the design of digital controllers are (e.g., see $[2,10,5])$ :

1. design based on a continuous-time plant model plus a controller discretization (the CTD method);

2. design based on the exact or approximate (such as Euler) discrete-time plant model ignoring intersample behavior (respectively, the DTD method and the approximate DTD method);

3. design based on the sampled-data model of the plant which takes into account the inter-sample behavior in the design procedure (the SDD method).

In principle, the DTD method is more straightforward for linear systems than for nonlinear systems. Indeed, for linear systems we can write down an explicit, exact discrete-time model while typically for nonlinear systems we cannot. Moreover, the exact discrete-time model of a linear system is linear while

\footnotetext{
*This work was done while the author was visiting Electrical and Computer Engineering Department, University of California, Santa Barbara, CA. Research supported by National Science Foundation under grant ECS-9812346. Email: d.nesic@ee.mu.oz.au

${ }^{\dagger}$ This work was supported in part by AFOSR grant F49620-98-1-0087 and in part by National Science Foundation National Science Foundation under grant ECS-9896140. Email: teel@ece.ucsb.edu

${ }^{\ddagger}$ Research supported by National Science Foundation under grant ECS-9812346. Email: petar@ece.ucsb.edu
} 
the exact discrete-time model for a nonlinear systems does not usually preserve important structures of the nonlinear systems, like affine controls for example. Consequently, for nonlinear systems it is unusual to assume knowledge of the exact discrete-time model whereas this assumption is made in most of classical linear literature. However, often due to our inability to exactly compute the matrix exponential that generates the exact discrete-time model we use its approximation and hence we actually use approximate DTD method most of the time, even for linear systems. The results of this paper illustrate that if one is not careful about choosing the approximate model and the controller, then even for linear systems it is possible to design a controller that stabilizes the approximate discrete-time model (for all small sampling periods) but destabilizes the exact discrete-time model (for all small sampling periods). These examples motivate a careful investigation of the approximate DTD method even in the linear context although our work was originally motivated only by nonlinear sampled-data systems.

The majority of work for nonlinear sampled-data systems uses the CTD method, see [1, 3, 7, 16, 17, $25,24]$. For results related to the approximate DTD method see $[4,6,8,12,15]$ and for approximate discretization procedures see $[13,22]$. Due to the complexity of the problem, results on the SDD method for nonlinear systems are scarce. For details on the SDD method for linear systems see [2].

The main question in the CTD, DTD and approximate DTD methods is whether or not the designed controllers stabilize (and in what sense) the original continuous-time plant. This question has been addressed and answered for the CTD method for nonlinear systems in $[1,3,16,17,25,24]$ and for the DTD method in [14]. We are concerned with the above question for the approximate DTD method for nonlinear systems.

We emphasize that the majority of nonlinear discrete-time literature is based on the assumption, which is unrealistic in general, that the exact discrete-time model of the plant is known. Hence, a fixed model is used for a design of a fixed controller. On the other hand, if we use the approximate DTD method then the sampling period $T$ typically should be sufficiently small in order to have a small mismatch between the exact and approximate models. So, we have to deal with a family of approximate discrete-time plant models for which a family of stabilizing controllers are designed.

Despite all the above difficulties one faces in the approximate DTD method for nonlinear systems, there is a strong motivation for pursuing this approach since it deals with the issue of sampling naturally and effectively, enabling direct theoretical investigations of the effect of sampling on the key system theoretic properties. As a result, the approximate DTD method may yield better results than the CTD method. For instance, a larger domain of attraction may be obtained with the approximate DTD method than with the CTD method for the same sampling period (e.g., see Example 1 in Section 4). One may argue that this follows from the fact that an approximate discrete-time model is better than the continuous-time model at approximating the exact discrete-time model. However, satisfactory results using the approximate DTD method can be obtained only if the approximate discrete-time model and the designed controller satisfy appropriate conditions (cf. Example 3 of Section 4).

Different variations of the approximate DTD method have been addressed in the adaptive control literature $[4,6,12]$. However, in these references, the plant, controller and discretization usually have a specific structure. We work in a slightly different setting but also use more generic and weaker conditions on the plant and controller structures. For a related result on regulation under sampling see [1].

We present conditions under which the controllers which stabilize an approximate discrete-time model of a nonlinear plant for $T \in] 0, T^{*}$ [ also stabilize the exact discrete-time model of the plant for $T \in] 0, \hat{T}\left[, \hat{T} \leq T^{*}\right.$. This justifies using the approximate DTD method under relatively weak conditions. The result is applicable to a wide range of plants, controllers and approximate discretization techniques. We will present two different results. One result (Theorem 2) assumes only that the trajectories of the approximate model remain relatively close to the trajectories of the exact model over one step (see Definition 1) and assumes for the family of approximate models the existence of a family of Lyapunov functions with certain properties that are uniform in the sampling period (see Definition 4). The other result (Theorem 1) assumes that the trajectories of the approximate model remain close to the trajectories of the exact model over many steps (see Definition 2) and assumes the trajectories of the approximate model exhibit stability that is partially uniform in the sampling period (see Definition $3)$. 
Our results are closely related to results in the numerical analysis literature on lower semi-continuity of attractors for numerical integration routines (see [23, Section 7.7] and the references therein) and in many places we have adapted, to our sampled-data nonlinear control problem, conditions and proof techniques traditionally used in the numerical analysis literature.

The paper is organized as follows. In Section 2.1 we present notation and define the problem setting. In Section 2.2 we define and clarify the notions of solution closeness, between exact and approximate models, that will be used in our main results. In Section 2.3 we define the types of stability that we are interested in and that we will assume for the family of approximate plant models. We define stability concepts in terms of bounds on trajectories and in terms of Lyapunov functions and we relate the two characterizations. Our main results are stated in Section 3. Several examples that illustrate our main results are presented in Section 4.

\section{Preliminaries}

\subsection{Problem setting and notation}

A function $\gamma: \mathbb{R}_{\geq 0} \rightarrow \mathbb{R}_{\geq 0}$ is of class- $\mathcal{K}(\gamma \in \mathcal{K})$ if it is continuous, strictly increasing and $\gamma(0)=0$. It is of class- $\mathcal{K}_{\infty}$ if it is of class- $\mathcal{K}$ and is unbounded. Functions of class- $\mathcal{K}_{\infty}$ are invertible. A function $\beta: \mathbb{R}_{\geq 0} \times \mathbb{R}_{\geq 0} \rightarrow \mathbb{R}_{\geq 0}$ is of class- $\mathcal{K} \mathcal{L}$ if $\beta(\cdot, t)$ is of class- $\mathcal{K}$ for each $t \geq 0$ and $\beta(s, \cdot)$ is decreasing to zero for each $s>0$. Sets of real and natural numbers are respectively denoted as $\mathbb{R}$ and $\mathbb{N}$. The Euclidean norm of a vector $x$ is denoted as $|x|$. Given a set $B \subset \mathbb{R}^{n}$, its $\epsilon$ neighborhood is denoted as $N(B, \epsilon):=\left\{x: \inf _{z \in B}|x-z| \leq \epsilon\right\}$.

Consider the nonlinear continuous-time control system:

$$
\dot{x}=f(x, u) \quad x(0)=x_{\circ}
$$

where $x \in \mathbb{R}^{n}, u \in \mathbb{R}^{m}$ are respectively the state and control vectors. The function $f$ is assumed to be such that, for each initial condition and each constant control, there exists a unique solution defined on some (perhaps bounded) interval of the form $[0, \tau)$. The control is taken to be a piecewise constant signal $u(t)=u(k T)=: u(k), \forall t \in[k T,(k+1) T[, k \in \mathbb{N}$ where $T>0$ is a sampling period. We assume that the state measurements $x(k):=x(k T)$ are available at sampling instants $k T, k \in \mathbb{N}$. The sampling period $T$ is assumed to be a design parameter which can be arbitrarily assigned (in practice, the sampling period $T$ is fixed and our results could be used to determine if it is suitably small).

Suppose that we want to design a control law for the plant (1) using the approximate DTD method. The controller will be implemented digitally using a sampler and zero order hold element. As a first step in this direction we need to specify a discrete-time model of the plant (1), which describes the behavior of the system at sampling instants. We consider the difference equations corresponding to the exact plant model and its approximation respectively:

$$
\begin{aligned}
& x(k+1)=F_{T}^{e}(x(k), u(k)) \\
& x(k+1)=F_{T}^{a}(x(k), u(k))
\end{aligned}
$$

which are parameterized with the sampling period $T$. We emphasize that $F_{T}^{e}$ is not known in most cases. We will think of $F_{T}^{e}$ and $F_{T}^{a}$ as being defined globally for all small $T$ even though the initial value problem (1) may exhibit finite escape times. We do this by defining $F_{T}^{e}$ arbitrarily for pairs $\left(x_{k}, u_{k}\right)$ corresponding to finite escapes and noting that such points correspond only to points of arbitrarily large norm as $T \rightarrow 0$, at least when $f$ is locally bounded. So, the behavior of $F_{T}^{e}$ will reflect the behavior of (1) as long as $\left(x_{k}, u_{T}\left(x_{k}\right)\right)$ remains bounded with a bound that is allowed to grow as $T \rightarrow 0$. This is consistent with our main results that guarantee practical asymptotic stability that is semiglobal in the sampling period, i.e., as $T \rightarrow 0$ the set of points from which convergence to an arbitrarily small ball is guaranteed to contain an arbitrarily large neighborhood of the origin.

In general, one needs to use small sampling periods $T$ since the approximate plant model is a good approximation of the exact model typically only for small $T$. It is clear then that we need to be able to 
obtain a controller $u_{T}(x)$ which is, in general, parameterized by $T$ and which is defined for all small $T$. For simplicity, we consider only static state feedback controllers.

For a fixed $T>0$, consider systems (2), (3) with a given controller $u(k)=u_{T}(x(k))$. We denote the state of the closed-loop system (2) (respectively (3)) with the given controller at time step $k$ that starts from $x(0)$ as $x^{e}(k, x(0))$ or $x_{k}^{e}$ (respectively $x^{a}(k, x(0))$ or $\left.x_{k}^{a}\right)$. We introduce the error:

$$
\varepsilon_{k}(\xi, z):=x^{e}(k, \xi)-x^{a}(k, z),
$$

and also use the notation $\varepsilon_{k}(\xi):=\varepsilon_{k}(\xi, \xi)=x^{e}(k, \xi)-x^{a}(k, \xi)$.

\subsection{Consistency of approximate model with exact model}

In our results (see Theorems 1 and 2), we will make a stability assumption on the family of closed-loop approximate plant models and will draw a conclusion about stability of the family of closed-loop exact plant models by invoking assumptions about the closeness of solutions between the two families. In this section we define the properties we will use.

\subsubsection{One-step consistency}

The first type of closeness we will use is characterized in the following definition. It guarantees that the error between solutions starting from the same initial condition is small, over one step, relative to the size of the step. The terminology we use is based on that used in the numerical analysis literature.

Definition 1 The family $\left(u_{T}, F_{T}^{a}\right)$ is said to be one-step consistent with $\left(u_{T}, F_{T}^{e}\right)$ if, for each compact set $\mathcal{X} \subset \mathbb{R}^{n}$, there exist a function $\rho \in \mathcal{K}_{\infty}$ and $T^{*}>0$ such that, for all $x \in \mathcal{X}$ and $\left.T \in\right] 0, T^{*}[$, we have

$$
\left|F_{T}^{e}\left(x, u_{T}(x)\right)-F_{T}^{a}\left(x, u_{T}(x)\right)\right| \leq T \rho(T) .
$$

A sufficient condition for one-step consistency is the following:

\section{Lemma 1 If}

1. $\left(u_{T}, F_{T}^{a}\right)$ is one-step consistent with $\left(u_{T}, F_{T}^{\text {Euler }}\right)$ where $F_{T}^{\text {Euler }}(x, u):=x+T f(x, u)$,

2. for each compact set $\mathcal{X} \subset \mathbb{R}^{n}$ there exist $\rho \in \mathcal{K}_{\infty}, M>0, T^{*}>0$ such that, for all $\left.T \in\right] 0, T^{*}[$ and all $x, y \in \mathcal{X}$

$$
\begin{aligned}
& \text { (a) }\left|f\left(y, u_{T}(x)\right)\right| \leq M, \\
& \text { (b) }\left|f\left(y, u_{T}(x)\right)-f\left(x, u_{T}(x)\right)\right| \leq \rho(|y-x|) \text {, }
\end{aligned}
$$

then $\left(u_{T}, F_{T}^{a}\right)$ is one-step consistent with $\left(u_{T}, F_{T}^{e}\right)$.

Proof. Let $\mathcal{X}_{1} \subset \mathbb{R}^{n}$ be an arbitrary compact set. Using the set $\mathcal{X}:=N\left(\mathcal{X}_{1}, 1\right)$, let the second assumption of the lemma generate $T^{*}>0, M>0$ and $\rho \in \mathcal{K}_{\infty}$. Let $T_{1}^{*}:=\min \left\{T^{*}, 1 / M\right\}$. It follows from assumption $2 \mathrm{a}$ of the lemma that, for each $x \in \mathcal{X}_{1}$ and all $\left.t \in\right] 0, T_{1}^{*}[$, the solution $\phi(t, x)$ of

$$
\dot{\phi}(t, x)=f\left(\phi(t, x), u_{T}(x)\right) \quad \phi(0, x)=x
$$

belongs to $\mathcal{X}$ and satisfies $|\phi(t, x)-x| \leq M t$. It then follows from assumption $2 \mathrm{~b}$ of the lemma that, for all $x \in \mathcal{X}_{1}$ and all $\left.T \in\right] 0, T_{1}^{*}[$,

$$
\left|\int_{0}^{T}\left[f\left(\phi(t, x), u_{T}(x)\right)-f\left(x, u_{T}(x)\right)\right] d t\right| \leq \int_{0}^{T} \rho(|\phi(t, x)-x|) d t \leq T \rho(M T) .
$$

Since

$$
F_{T}^{e}\left(x, u_{T}(x)\right)=x+T f\left(x, u_{T}(x)\right)+\int_{0}^{T}\left[f\left(\phi(t, x), u_{T}(x)\right)-f\left(x, u_{T}(x)\right)\right] d t
$$

the result follows from (7) and the first assumption of the lemma. Q.E.D. 
Remark 1 A special case where condition $2 \mathrm{~b}$ of the lemma holds is when $f(\cdot, u)$ is locally Lipschitz uniformly in $u$. Note that the function $u_{T}(x)$ does not need to be continuous for $\left(u_{T}, F_{T}^{a}\right)$ to be one-step consistent with $\left(u_{T}, F_{T}^{e}\right)$. A control system with a discontinuous feedback is considered in Example 2 of Section 4 .

\subsubsection{Multi-step consistency}

The second type of closeness we will use is characterized in terms of the functions $F_{T}^{e}, F_{T}^{a}$ and $u_{T}(x)$ in the next definition. It will guarantee (see Lemma 2) that the error between solutions starting from the same initial condition is small over multiple steps corresponding "continuous-time" intervals with length of order one.

Definition 2 The family $\left(u_{T}, F_{T}^{a}\right)$ is said to be multi-step consistent with $\left(u_{T}, F_{T}^{e}\right)$ if, for each $L>0$, $\eta>0$ and each compact set $\mathcal{X} \subset \mathbb{R}^{n}$, there exist a function $\alpha: \mathbb{R}_{\geq 0} \times \mathbb{R}_{\geq 0} \rightarrow \mathbb{R}_{\geq 0} \cup\{\infty\}$ and $T^{*}>0$ such that, for all $T \in] 0, T^{*}[$ we have

$$
\{x, z \in \mathcal{X},|x-z| \leq \delta\} \quad \Longrightarrow \quad\left|F_{T}^{e}\left(x, u_{T}(x)\right)-F_{T}^{a}\left(z, u_{T}(z)\right)\right| \leq \alpha(\delta, T)
$$

and

$$
k \leq L / T \quad \Longrightarrow \quad \alpha^{k}(0, T):=\overbrace{\alpha(\cdots \alpha(\alpha}^{k}(0, T), T) \cdots, T) \leq \eta .
$$

In terms of trajectory error over "continuous-time" intervals with length of order one, multi-step consistency gives the following:

Lemma 2 If $\left(u_{T}, F_{T}^{a}\right)$ is multi-step consistent with $\left(u_{T}, F_{T}^{e}\right)$ then for each compact set $\mathcal{X} \subset \mathbb{R}^{n}, L>0$ and $\eta>0$ there exists $\hat{T}>0$ such that, if $T$ and $\xi$ satisfy

$$
T \in] 0, \hat{T}\left[, \quad x_{k}^{a}(\xi) \in \mathcal{X} \quad \forall k: k T \in[0, L],\right.
$$

then

$$
\left|\varepsilon_{k}(\xi)\right| \leq \eta \quad \forall k: k T \in[0, L]
$$

Proof. Define $\overline{\mathcal{X}}:=N(\mathcal{X}, \eta)$. Since $\left(u_{T}, F_{T}^{a}\right)$ is multi-step consistent with $\left(u_{T}, F_{T}^{e}\right)$, there exist a function $\alpha(\cdot, \cdot)$ and a strictly positive real number $T^{*}$ such that (9) and (10) are satisfied for the triple $(\overline{\mathcal{X}}, L, \eta)$. We now prove the result by induction. First we have $\left|\varepsilon_{0}(\xi)\right|=0 \leq \alpha^{1}(0, T) \leq \eta$. Next, suppose $\left|\varepsilon_{k}(\xi)\right| \leq \alpha^{k}(0, T) \leq \eta$ and $(k+1) T \in[0, L]$. Since $x_{k}^{a}(\xi) \in \mathcal{X}$, it follows from the definition of $\overline{\mathcal{X}}$ that $x_{k}^{a}(\xi), x_{k}^{e}(\xi) \in \overline{\mathcal{X}}$. It then follows from $(9)$ that $\left|\varepsilon_{k+1}(\xi)\right| \leq \alpha^{k+1}(0, T)$. Since $(k+1) T \in[0, L]$ it follows from (10) that $\alpha^{k+1}(0, T) \leq \eta$. Q.E.D.

Returning to Definition 2, we note that $\left(u_{T}, F_{T}^{e}\right)$ is multi-step consistent with itself. This follows by noting that when $F_{T}^{a}=F_{T}^{e}$ we can take $\alpha(0, T)=0$ and $\alpha(\delta, T)=2 \sup _{x \in \mathcal{X}}\left|F_{T}^{e}\left(x, u_{T}(x)\right)\right|$. Another, more interesting, sufficient condition for multi-step consistency is given in the following:

Lemma 3 If, for each compact set $\mathcal{X} \subset \mathbb{R}^{n}$, there exist $K>0, \rho \in \mathcal{K}_{\infty}$ and $T^{*}>0$ such that for all $T \in] 0, T^{*}[$ and all $x, z \in \mathcal{X}$ we have

$$
\left|F_{T}^{e}\left(x, u_{T}(x)\right)-F_{T}^{a}\left(z, u_{T}(z)\right)\right| \leq(1+K T)|x-z|+T \rho(T)
$$

then $\left(u_{T}, F_{T}^{a}\right)$ is multi-step consistent with $\left(u_{T}, F_{T}^{e}\right)$.

Proof. Let $\mathcal{X} \subset \mathbb{R}^{n}, L>0$ and $\eta>0$ be given. From the assumption of the lemma, let $\mathcal{X}$ generate $K>0, \rho \in \mathcal{K}_{\infty}$ and $T_{1}^{*}>0$. Define

$$
\begin{aligned}
\alpha(\delta, T) & :=(1+K T) \delta+T \rho(T) \\
T^{*} & :=\min \left\{T_{1}^{*}, \rho^{-1}\left(\frac{\eta K}{\exp (K L)-1}\right)\right\} .
\end{aligned}
$$


With these definitions, the condition (9) is satisfied. Also note that

$$
\alpha^{k}(0, T)=T \rho(T) \sum_{i=0}^{k-1}(1+K T)^{i}=\frac{\rho(T)}{K}\left[(1+K T)^{k}-1\right] \leq \frac{\rho(T)}{K}[\exp (k K T)-1]
$$

and so (10) is satisfied. Q.E.D.

Remark 2 Relative to the one-step consistency condition, the condition of Lemma 3 is guaranteed by one-step consistency plus the following type of Lipschitz condition on either the family $\left(u_{T}, F_{T}^{e}\right)$ or the family $\left(u_{T}, F_{T}^{a}\right)$ :

for each compact set $\mathcal{X} \subset \mathbb{R}^{n}$ there exist $K>0$ and $T^{*}>0$ such that for all $x, z \in \mathcal{X}$ and all $\left.T \in\right] 0, T^{*}[$,

$$
\left|F_{T}\left(x, u_{T}(x)\right)-F_{T}\left(z, u_{T}(z)\right)\right| \leq(1+K T)|x-z| .
$$

This condition is guaranteed for $F_{T}^{e}$ when $f(x, u)$ and $u_{T}(x)$ are locally Lipschitz (uniformly in small $T$ ). Note that no continuity assumptions on $u_{T}(x)$ were made in Lemma 1 to guarantee one-step consistency. The condition given in Lemma 3 for multi-step consistency is similar to conditions used in the numerical analysis literature (e.g., see conditions (i) and (iii) of Assumption 6.1.2 in [23, pg.429]).

Remark 3 One-step and multi-step consistency do not imply each other and this is one motivation for developing different stability theorems that rely on one of these properties. Example 2 in Section 4 shows that multi-step consistency may not hold when one-step consistency does hold. That one-step consistency may not hold when multi-step consistency does hold can be seen from the plant $\dot{x}=x+u$ with Euler approximation $x(k+1)=x(k)+T(x(k)+u(k))=F_{T}^{a}(x(k), u(k))$ and controller $u_{T}(x)=-\left(\frac{1}{T}+1\right) x$. The exact discrete-time model is $x(k+1)=e^{T} x(k)+\left(e^{T}-1\right) u(k)$ and we have $F_{T}^{a}\left(x, u_{T}(x)\right) \equiv 0$ and $F_{T}^{e}\left(x, u_{T}(x)\right)=\left(1-\frac{e^{T}-1}{T}\right) x$. Since, for $x$ in a compact set, $F_{T}^{e}\left(x, u_{T}(x)\right)$ is of order $T$ we do not have one-step consistency. On the other hand, it follows from $F_{T}^{a}\left(x, u_{T}(x)\right) \equiv 0$ and the fact that $F_{T}^{e}\left(x, u_{T}(x)\right)$ is of order $T$ that we do have multi-step consistency. Indeed, for each compact set $\mathcal{X} \subset \mathbb{R}$ and each $\eta>0$ there exist strictly positive numbers $K, T^{*}$ such that, for all $\left.x, z \in \mathcal{X}, T \in\right] 0, T^{*}[, k \geq 0$,

$$
\left|F_{T}^{e}\left(x, u_{T}(x)\right)-F_{T}^{a}\left(z, u_{T}(z)\right)\right|=\left|F_{T}^{e}\left(x, u_{T}(x)\right)\right| \leq K T:=\alpha(\delta, T)=\alpha^{k}(0, T) \leq \eta .
$$

\subsection{Stability properties}

The goal of our paper is to give conditions on the family $\left(u_{T}, F_{T}^{a}\right)$ that guarantee asymptotic stability for the family $\left(u_{T}, F_{T}^{e}\right)$. In Example 3 of Section 4 we will see that it is not enough to assume simply that each member of the family $\left(u_{T}, F_{T}^{a}\right)$ is asymptotically stable (at least for small $T$ ). Instead, we will impose partial uniformity of the stability property over all small $T$. For that, we make the following definitions:

Definition 3 Let $\beta \in \mathcal{K} \mathcal{L}$ and let $N \subset \mathbb{R}^{n}$ be an open (not necessarily bounded) set containing the origin.

1. The family $\left(u_{T}, F_{T}\right)$ is said to be $(\beta, N)$-stable if there exists $T^{*}>0$ such that for each $\left.T \in\right] 0, T^{*}[$, the solutions of the system

$$
x(k+1)=F_{T}\left(x(k), u_{T}(x(k))\right)
$$

satisfy

$$
|x(k, x(0))| \leq \beta(|x(0)|, k T), \quad \forall x(0) \in N, k \geq 0 .
$$


2. The family $\left(u_{T}, F_{T}\right)$ is said to be $(\beta, N)$-practically stable if for each $R>0$ there exists $T^{*}>0$ such that for each $T \in] 0, T^{*}[$ the solutions of (17) satisfy:

$$
|x(k, x(0))| \leq \beta(|x(0)|, k T)+R, \forall x(0) \in N, k \geq 0 .
$$

Remark 4 It is standard to use $\mathcal{K} \mathcal{L}$ functions to characterize uniform global asymptotic stability for differential equations (e.g., see Proposition 2.5 in [11]). The stability properties defined above have the property that the solutions $x^{a}(k, x(0))$ are allowed to "slow down" in discrete-time as $T$ is decreased. However, the upper bound on the rate of decrease of $\left|x^{a}(k, x(0))\right|$ is uniform if $k T$ is thought of as a "continuous time" parameter. Moreover, the overshoots are uniform in $T$.

Remark 5 If we can show that the family $\left(u_{T}, F_{T}^{e}\right)$ is $(\beta, N)$-practically stable and if we can appropriately bound the intersample behavior for $\dot{x}=f(x, u)$ for sufficiently small $T$ then the main results of [14] can be used to conclude that the sampled-data system is uniformly practically asymptotically stable for sufficiently small $T$. A sufficient condition for being able to bound the inter-sample behavior appropriately is that condition 2 (a) of Lemma 1 holds.

An equivalent Lyapunov formulation of $\left(\beta, \mathbb{R}^{n}\right)$-stability is the following (local versions can also be formulated but are more tedious to state because of the need to keep track of basins of attraction):

Lemma 4 The following statements are equivalent:

1. There exists $\beta \in \mathcal{K} \mathcal{L}$ such that the family $\left(u_{T}, F_{T}\right)$ is $\left(\beta, \mathbb{R}^{n}\right)$-stable.

2. There exist $T^{*}>0, \alpha_{1}, \alpha_{2} \in \mathcal{K}_{\infty}, \alpha_{3} \in \mathcal{K}$ and for each $\left.T \in\right] 0, T^{*}\left[, V_{T}: \mathbb{R}^{n} \rightarrow \mathbb{R}_{\geq 0}\right.$ such that $\left.\forall x \in \mathbb{R}^{n}, \forall T \in\right] 0, T^{*}[$ we have:

$$
\begin{array}{r}
\alpha_{1}(|x|) \leq V_{T}(x) \leq \alpha_{2}(|x|), \\
V_{T}\left(F_{T}\left(x, u_{T}(x)\right)\right)-V_{T}(x) \leq-T \alpha_{3}(|x|), .
\end{array}
$$

\section{Proof.}

$(2) \Longrightarrow(1)$. See Remark 7 which follows the proof of Theorem 2.

$(1) \Longrightarrow(2)$. (This proof is based on the construction, for differential inclusions, given in [21].) According to [19, Proposition 7], given $\beta \in \mathcal{K} \mathcal{L}$ there exist class- $\mathcal{K}_{\infty}$ functions $\alpha_{1}$ and $\alpha_{2}$ such that

$$
\alpha_{1}(\beta(s, t)) \leq \alpha_{2}(s) e^{-3 t}
$$

Let $T^{*}>0$ be such that

$$
\left.T \leq 1-e^{-2 T} \quad \forall T \in\right] 0, T^{*}[
$$

We define

$$
V_{T}(\xi):=\sup _{k \geq 0} \alpha_{1}\left(\left|x_{T}(k, \xi)\right|\right) e^{2 k T}
$$

We then have

$$
V_{T}(\xi) \geq \alpha_{1}\left(\left|x_{T}(0, \xi)\right|\right)=\alpha_{1}(|\xi|)
$$

and, using (22) and the $\left(\beta, \mathbb{R}^{n}\right)$-stability assumption,

$$
V_{T}(\xi) \leq \sup _{k \geq 0} \alpha_{1}(\beta(|\xi|, k T)) e^{2 k T} \leq \sup _{k \geq 0} \alpha_{2}(|\xi|) e^{-k T}=\alpha_{2}(|\xi|) .
$$


This establishes (20). To establish (21) we note that (using the shorthand notation $F_{T}(\xi)=F_{T}\left(\xi, u_{T}(\xi)\right)$ )

$$
\begin{aligned}
V_{T}\left(F_{T}(\xi)\right) & =\sup _{k \geq 0} \alpha_{1}\left(\left|x_{T}\left(k, F_{T}(\xi)\right)\right|\right) e^{2 k T} \\
& =\sup _{k \geq 1} \alpha_{1}\left(\left|x_{T}(k, \xi)\right|\right) e^{2(k-1) T} \\
& \leq \sup _{k \geq 0} \alpha_{1}\left(\left|x_{T}(k, \xi)\right|\right) e^{2 k T} e^{-2 T} \\
& =V_{T}(\xi) e^{-2 T} \\
& =V_{T}(\xi)-V_{T}(\xi)\left[1-e^{-2 T}\right] .
\end{aligned}
$$

Then, using (23) and (25), this established (21) with $\alpha_{3}(s)=\alpha_{1}(s)$. Q.E.D.

In our first main result (Theorem 1) we will show that if the family $\left(u_{T}, F_{T}^{a}\right)$ is $(\beta, N)$-stable and multi-step consistent with $\left(u_{T}, F_{T}^{e}\right)$ then the family $\left(u_{T}, F_{T}^{e}\right)$ is $(\beta, N)$-practically stable. We will also show (in Theorem 2) that the multi-step consistency assumption can be changed to a one-step consistency assumption when $(\beta, N)$-stability is formulated in terms of a family of Lyapunov functions satisfying $(20),(21)$ and with an extra local Lipschitz condition that is uniform in small $T$.

Definition 4 The family $\left(u_{T}, F_{T}\right)$ is said to be equi-globally asymptotically stable (EGAS) by equiLipschitz Lyapunov functions if the second statement of Lemma 4 holds and, moreover, for each compact set $\mathcal{X} \subset \mathbb{R}^{n} \backslash\{0\}$ there exist $M>0$ and $T^{*}>0$ such that, for all $x, z \in \mathcal{X}$ and all $\left.T \in\right] 0, T^{*}[$,

$$
\left|V_{T}(x)-V_{T}(z)\right| \leq M|x-z| .
$$

\section{Main Results}

In this section we present conditions under which the approximate DTD method is a valid approach for the design of controllers for nonlinear discrete-time systems and, in turn, via the results from [14], a valid approach to design controllers for sampled-data systems.

\subsection{Stability from trajectory bounds and multi-step consistency}

Our first result is expressed in terms of trajectory bounds for $\left(u_{T}, F_{T}^{a}\right)$ and multi-step consistency:

Theorem 1 Let $\beta \in \mathcal{K} \mathcal{L}$ and let $N$ be a bounded neighborhood of the origin. If the family $\left(u_{T}, F_{T}^{a}\right)$ is:

A: multi-step consistent with $\left(u_{T}, F_{T}^{e}\right)$, and

B: $(\beta, N)$-stable,

then

C: the family $\left(u_{T}, F_{T}^{e}\right)$ is $(\beta, N)$-practically stable.

Proof: Let $R>0, \beta \in \mathcal{K} \mathcal{L}$ and the bounded neighborhood $N$ of the origin be given. We want to establish that there exists $\hat{T}>0$ such that for all $T \in] 0, \hat{T}[$, all $x(0) \in N$ and all $k \geq 0$ we have

$$
\left|x^{e}(k, x(0))\right| \leq \beta(|x(0)|, k T)+R .
$$

Let $M=\sup _{\xi \in N}|\xi|$ and let $B$ be the closed ball of radius $\beta(M, 0)$. Let $\eta>0$ be such that the closed ball of radius $2 \eta$ is contained in $N$ and $\beta(2 \eta, 0)<R / 2$. Such an $\eta$ exists since $N$ is a neighborhood of the origin and $\beta(s, 0) \in \mathcal{K}$. It also follows from Assumption B of the theorem and (18) with $k=0$ that $\beta(s, 0) \geq s$ so that $2 \eta<R / 2$. Let $L>0$ be such that $\beta(M, t) \leq \eta$ for all $t \geq L-1$. Using the fact that the family $\left(u_{T}^{a}, F_{T}^{a}\right)$ is $(\beta, N)$-stable, so that $x_{k}^{a}(\xi) \in B$ for all $\xi \in N$ and all $k \geq 0$, and Assumption A 
and applying Lemma 2 with the triple $(B, L, \eta)$, there exists $\hat{T}>0$ such that $x(0) \in N, T \in] 0, \hat{T}[$ and $k: k T \in[0, L]$ imply

$$
\left|\varepsilon_{k}(x(0))\right| \leq \eta
$$

Thus we have, for such $(T, k, x(0))$,

$$
\left|x^{e}(k, x(0))\right| \leq\left|x^{a}(k, x(0))\right|+\left|\varepsilon_{k}(x(0))\right| \leq \beta(|x(0)|, k T)+\eta .
$$

Since $\eta<R / 4,(29)$ holds for all $x(0) \in N$, all $T \in] 0, \hat{T}[$ and all $k$ such that $k T \in[0, L]$. It remains to establish the result for larger $k$. Without loss of generality, assume $\hat{T} \leq 1$. Define $k_{T}^{*}=$ $\max \{k \in \mathbb{N}: k T \leq L\}$. Note that $k_{T}^{*}$ is such that, for all $\left.T \in\right] 0, \hat{T}[$,

$$
k_{T}^{*} T>L-T>L-1 .
$$

Thus, using the definition of $L$ and (31), we get

$$
\left|x^{e}\left(k_{T}^{*}, x(0)\right)\right| \leq 2 \eta .
$$

Now consider those $k$ such that $k \in\left[k_{T}^{*}, 2 k_{T}^{*}\right]$. We have, using time-invariance, (30), (33) and the fact that the ball of radius $2 \eta$ is contained in $N$, we get

$$
\begin{aligned}
\left|x^{e}(k, x(0))\right| & =\left|x^{e}\left(k-k_{T}^{*}, x^{e}\left(k_{T}^{*}, x(0)\right)\right)\right| \\
& \leq\left|x^{a}\left(k-k_{T}^{*}, x^{e}\left(k_{T}^{*}, x(0)\right)\right)\right|+\left|\varepsilon_{k-k_{T}^{*}}\left(x^{e}\left(k_{T}^{*}, x(0)\right)\right)\right| \\
& \leq \beta\left(2 \eta,\left(k-k_{T}^{*}\right) T\right)+\eta
\end{aligned}
$$

from which it follows that, for all $k \in\left[k_{T}^{*}, 2 k_{T}^{*}\right]$,

$$
\left|x^{e}(k, x(0))\right| \leq \beta(2 \eta, 0)+\eta \leq R / 2+R / 4 \leq R
$$

(which implies (29) ) and, using the definition of $k_{T}^{*}$ we have that

$$
\left|x^{e}\left(2 k_{T}^{*}, x(0)\right)\right| \leq \beta\left(2 \eta, k_{T}^{*} T\right)+\eta \leq 2 \eta .
$$

The result then follows by induction. Q.E.D.

Remark 6 In the $(\beta, N)$-stability assumption for the family $\left(u_{T}, F_{T}^{a}\right)$, if $N$ can be made arbitrarily large by taking $T$ sufficiently small (e.g., $N=\mathbb{R}^{n}$ ) then the family $\left(u_{T}, F_{T}^{e}\right)$ exhibits semiglobal practical (in the sampling period $T$ ) asymptotic stability.

\subsection{Stability from Lyapunov functions and one-step consistency}

Our second result is expressed in terms of a family of Lyapunov functions for $\left(u_{T}, F_{T}^{a}\right)$ and one-step consistency. It has some relations to the proof technique used to establish the main result of [3]. For simplicity we will only formulate the global result. Nonglobal results and results for stability of sets other than the origin can also be established with the same proof technique.

\section{Theorem 2 If}

A1: $\left(u_{T}, F_{T}^{a}\right)$ is one-step consistent with $\left(u_{T}, F_{T}^{e}\right)$, and

B1: $\left(u_{T}, F_{T}^{a}\right)$ is EGAS by equi-Lipschitz Lyapunov functions

then 
C1: there exists $\beta \in \mathcal{K} \mathcal{L}$ such that, for each bounded neighborhood $N$ of the origin, the family $\left(u_{T}, F_{T}^{e}\right)$ is $(\beta, N)$-practically stable.

Proof. We claim that, for each pair of strictly positive real numbers $(d, D)$, there exists $T^{*}>0$ such that, for all $T \in] 0, T^{*}$, we have

$$
\left\{\max \left\{V_{T}\left(F_{T}^{e}\left(x, u_{T}(x)\right)\right), V_{T}(x)\right\} \geq d\right\} \quad \Longrightarrow \quad V_{T}\left(F_{T}^{e}\left(x, u_{T}(x)\right)\right)-V_{T}(x) \leq-\frac{T}{2} \alpha_{3}(|x|)
$$

For now we take this to be true. In the rest of the proof we will use the notation $V_{T}(k)$ in place of $V_{T}\left(x_{k}^{e}\right)$. Let $N$ be an arbitrary bounded neighborhood of the origin, let

$$
D:=\sup _{x \in N} \alpha_{1}^{-1} \circ \alpha_{2}(|x|)
$$

and let $d$ be an arbitrary real number in the interval $] 0, \alpha_{1}(D)[$. Using this pair $(d, D)$ in the claim, it follows that there exists $T^{*}>0$ such that, for all $\left.T \in\right] 0, T^{*}[$ and all $x(0) \in N$ we have

$$
V_{T}(k) \leq \max \left\{V_{T}(0), d\right\}
$$

and

$$
\max \left\{V_{T}(k+1), V_{T}(k)\right\} \geq d \quad \Longrightarrow \quad V_{T}(k+1)-V_{T}(k) \leq-\frac{T}{2} \alpha_{3}\left(\alpha_{2}^{-1}\left(V_{T}(k)\right)\right)=:-T \alpha\left(V_{T}(k)\right)
$$

The relation (39) follows by induction. Indeed, (39) clearly holds for $k=0$ and then (39), (20) and the definitions of $d$ and $D$ imply

$$
\left|x_{k}^{e}\right| \leq \max \left\{\alpha_{1}^{-1}\left(V_{T}(0)\right), \alpha_{1}^{-1}(d)\right\} \leq D
$$

and so either $V_{T}(k+1) \geq d$ which, from $(37)$ and $(41)$, implies $V_{T}(k+1) \leq V_{T}(k)$ or else $V_{T}(k+1) \leq d$ so that, in either case, $(39)$ holds for $V_{T}(k+1)$ as well. With $(37),(41)$ and $(20)$, the relation (40) is immediate.

Introduce $y_{T}(t):=V_{T}(k)+\left(\frac{t}{T}-k\right)\left(V_{T}(k+1)-V_{T}(k)\right), \forall t \in\left[k T,(k+1) T\left[, k \geq 0\right.\right.$. Then $y_{T}(t)$ is a continuous, non-negative, piecewise linear function of "time" $t$ and hence it is absolutely continuous with derivative given, for almost all $t$, by

$$
\dot{y}_{T}(t)=\frac{V_{T}(k+1)-V_{T}(k)}{T}, \quad t \in[k T,(k+1) T[
$$

Using the definition of $y_{T}(t)$ and (40), we have

$$
\begin{aligned}
\left\{y_{T}(t) \geq d, t \in[k T,(k+1) T[\}\right. & \Longrightarrow \max \left\{V_{T}(k+1), V_{T}(k)\right\} \geq d \\
& \Longrightarrow V_{T}(k+1)-V_{T}(k) \leq-T \alpha\left(V_{T}(k)\right) \\
& \Longrightarrow y_{T}(t) \leq V_{T}(k)
\end{aligned}
$$

and so, combining with (42), we can write for almost all $t$ that:

$$
\begin{aligned}
y_{T}(t) \geq d \quad \Longrightarrow \quad \dot{y}_{T}(t) & \leq-\alpha\left(V_{T}(k)\right), \quad k: t \in[k T,(k+1) T[ \\
& \leq-\alpha\left(y_{T}(t)\right) .
\end{aligned}
$$

It follows from the arguments in $\left[18\right.$, Section VI] that there exists $\beta_{1} \in \mathcal{K} \mathcal{L}$ that is determined by $\alpha=\alpha_{3} \circ \alpha_{2}^{-1}$, i.e., independent of $T$ and $d$, such that

$$
y_{T}(t) \leq \max \left\{\beta_{1}\left(y_{T}(0), t\right), d\right\}, \forall t \geq 0
$$


This implies, using $V_{T}(k)=y_{T}(k)$ with $t=k T, y_{T}(0)=V_{T}(0)$, that

$$
V_{T}(k) \leq \max \left\{\beta_{1}\left(V_{T}(0), k T\right), d\right\}, k \geq 0 .
$$

By using (20) we obtain:

$$
|x(k)| \leq \alpha_{1}^{-1}\left(\beta_{1}\left(\alpha_{2}(|x(0)|), k T\right)\right)+\alpha_{1}^{-1}(d), \quad \forall x(0) \in N, k \geq 0 .
$$

Clearly $\beta(s, \tau):=\alpha_{1}^{-1}\left(\beta_{1}\left(\alpha_{2}(s), \tau\right)\right)$ is a class- $\mathcal{K} \mathcal{L}$ function that is independent of $T$ and $d$, and $d$ can be chosen arbitrarily small.

It remains to establish our initial claim in (37). Given the pair of strictly positive real numbers $(d, D)$, we make the definitions

$$
\begin{aligned}
\delta & :=\frac{1}{2} \alpha_{2}^{-1}\left(\frac{d}{2}\right) \\
\Delta & :=\alpha_{1}^{-1} \circ \alpha_{2}(D)+\frac{1}{2} \alpha_{2}^{-1}\left(\frac{d}{2}\right) .
\end{aligned}
$$

From Assumption B1, we let the set $\mathcal{X}:=\left\{z \in \mathbb{R}^{n}:|z| \in[\delta, \Delta]\right\}$ generate $M>0$ and $T_{1}^{*}>0$. From Assumption A1, we let the set $\mathcal{X}_{1}$, defined to be the closed ball of radius $D$, generate the function $\rho \in \mathcal{K}$ and $T_{2}^{*}>0$. We let $T_{3}^{*}>0$ be such that

$$
M \rho\left(T_{3}^{*}\right) \leq \frac{1}{2} \alpha_{3} \circ \alpha_{2}^{-1} \circ \alpha_{1}(\delta)
$$

and let $T_{4}^{*}>0$ be such that

$$
T_{4}^{*} \rho\left(T_{4}^{*}\right) \leq \frac{1}{2} \alpha_{2}^{-1}\left(\frac{d}{2}\right) .
$$

Then we take $T^{*}=\min \left\{T_{1}^{*}, T_{2}^{*}, T_{3}^{*}, T_{4}^{*}\right\}$.

Suppose $|x| \leq D$. From (20) this implies

$$
\left|F_{T}^{a}\left(x, u_{T}(x)\right)\right| \leq \alpha_{1}^{-1}\left(V_{T}\left(F_{T}^{a}\left(x, u_{T}(x)\right)\right) \leq \alpha_{1}^{-1}\left(V_{T}(x)\right) \leq \alpha_{1}^{-1} \circ \alpha_{2}(|x|) \leq \alpha_{1}^{-1} \circ \alpha_{2}(D) \leq \Delta .\right.
$$

From the choice of $T^{*}$ (in particular, the requirement on $T_{4}^{*}$ ) and (5), it follows that for all $\left.T \in\right] 0, T^{*}[$

$$
\left|F_{T}^{e}\left(x, u_{T}(x)\right)\right| \leq \alpha_{1}^{-1} \circ \alpha_{2}(D)+\frac{1}{2} \alpha_{2}^{-1}\left(\frac{d}{2}\right)=\Delta .
$$

Suppose $V_{T}\left(F_{T}^{e}\left(x, u_{T}(x)\right)\right) \geq d / 2$. From (20), this implies

$$
\left|F_{T}^{e}\left(x, u_{T}(x)\right)\right| \geq \alpha_{2}^{-1}\left(\frac{d}{2}\right) \geq \delta .
$$

From the choice of $T^{*}$ (in particular, the requirement on $T_{4}^{*}$ ) and (5), it follows that for all $\left.T \in\right] 0, T^{*}[$

$$
\left|F_{T}^{a}\left(x, u_{T}(x)\right)\right| \geq \frac{1}{2} \alpha_{2}^{-1}\left(\frac{d}{2}\right)=\delta
$$

and with (50) that

$$
|x| \geq \alpha_{2}^{-1} \circ \alpha_{1}(\delta) .
$$

From (50)-(54) and from the choice of $T^{*}$ we deduce that $|x| \leq D$ and $V_{T}\left(F_{T}^{e}\left(x, u_{T}(x)\right)\right) \geq d / 2$ imply

$$
\begin{aligned}
V_{T}\left(F_{T}^{e}\left(x, u_{T}(x)\right)\right)-V_{T}(x) & =V_{T}\left(F_{T}^{a}\left(x, u_{T}(x)\right)\right)-V_{T}(x)+V_{T}\left(F_{T}^{e}\left(x, u_{T}(x)\right)\right)-V_{T}\left(F_{T}^{a}\left(x, u_{T}(x)\right)\right) \\
& \leq-T \alpha_{3}(|x|)+M T \rho\left(T^{*}\right) \\
& \leq-\frac{T}{2} \alpha_{3}(|x|) .
\end{aligned}
$$


Next suppose $V_{T}\left(F_{T}^{e}\left(x, u_{T}(x)\right)\right) \leq d / 2$ and $V_{T}(x) \geq d$. We also know from $(20)$ that $V_{T}(x) \geq T \alpha_{3}(|x|)$. So, it follows that

$$
V_{T}\left(F_{T}^{e}\left(x, u_{T}(x)\right)\right)-V_{T}(x) \leq \frac{1}{2}\left(d-V_{T}(x)-V_{T}(x)\right) \leq-\frac{T}{2} \alpha_{3}(|x|) .
$$

This establishes the claim. Q.E.D.

Remark 7 The proof of $(2) \Longrightarrow(1)$ in Lemma 4 follows by taking $d=0$ and $D=\infty$ and following the preceding proof from inequality (39) to (46).

\section{Examples}

Now we present several examples. Example 1 illustrates our main theorems and shows a quantitative advantage in using the approximate DTD method over the CTD method. Example 2 illustrates Theorem 2 by giving an example where multi-step consistency does not hold but one-step consistency does hold and there is a suitable family of Lyapunov functions. Example 3 shows situation where each element of the family $\left(u_{T}, F_{T}^{a}\right)$ is globally exponentially stable with overshoots uniform in $T$ but where the family $\left(u_{T}, F_{T}^{e}\right)$ fails to be $(\beta, N)$-practically stable for any pair $(\beta, N)$. In reference to Theorem 1 , we use the notation $\overline{\mathbf{C}}$ for this situation. In Example $3, \overline{\mathbf{C}}$ occurs because the family $\left(u_{T}, F_{T}^{a}\right)$ is not multi-step (or one-step) consistent with $\left(u_{T}, F_{T}^{e}\right)$, i.e., $\overline{\mathbf{B}}(\overline{\mathbf{B 1}})$.

Example 1 Consider the plant:

$$
\dot{x}(t)=x^{3}(t)+u(t) .
$$

We design sampled-data nonlinear controllers based on the Euler approximation and a second order approximation of the plant, which are respectively given by:

$$
\begin{aligned}
& x(k+1)=x(k)+T\left(x^{3}(k)+u(k)\right)=: F_{T}^{a, 1}(x(k), u(k)) \\
& x(k+1)=x(k)+T\left(x^{3}(k)+u(k)\right)+\frac{3 T^{2}}{2} x^{2}(k)\left[x^{3}(k)+u(k)\right]=: F_{T}^{a, 2}(x(k), u(k))
\end{aligned}
$$

The controllers based on the approximations (58) and (59) that assign the following dynamics:

$$
x(k+1)=(1-T) x(k)
$$

to the closed-loop approximate systems are given, respectively, by:

$$
\begin{aligned}
& u_{T}^{1}(x(k))=-x(k)-x^{3}(k) \\
& u_{T}^{2}(x(k))=-\frac{x(k)+x^{3}(k)+\frac{3 T}{2} x^{5}(k)}{1+\frac{3 T}{2} x^{2}(k)} .
\end{aligned}
$$

A: We use Lemmas 1 and 3 with Remark 2 to check multi-step consistency. By definition, $\left(u_{T}^{1}, F_{T}^{a, 1}\right)$ is one-step consistent with $\left(u_{T}^{1}, F_{T}^{\text {Euler }}\right)$. Also, since $\left.\left|u_{T}^{\ell}(x)\right| \leq|x|+|x|^{3}+1.5|x|^{5}, \forall T \in\right] 0,1[, \ell=1,2$, $\left(u_{T}^{2}, F_{T}^{a, 2}\right)$ is one-step consistent with $\left(u_{T}^{2}, F_{T}^{\text {Euler }}\right)$. Moreover, with the bound on $\left|u_{T}^{\ell}(x)\right|$, it is easy to see that the second assumption of Lemma 1 also holds. So, from Lemma 1, the family $\left(u_{T}^{\ell}, F_{T}^{a, \ell}\right)$ is one-step consistent with $\left(u_{T}^{\ell}, F_{T}^{e}\right)$. Next, it is easy to check that the condition (16) is also satisfied so that, by Lemma 3 and Remark 2, the family $\left(u_{T}^{\ell}, F_{T}^{a, \ell}\right)$ is multi-step consistent with $\left(u_{T}^{\ell}, F_{T}^{e}\right)$.

B: It follows from the proof of (2) $\Longrightarrow(1)$ in Lemma 4 using $V_{T}(x)=|x|$ that both $\left(u_{T}^{\ell}, F_{T}^{a, \ell}\right), \ell=1,2$ are $(\beta, \mathbb{R})$-stable with $\beta(s, t):=s e^{-t}$.

C: We conclude from Theorem 1 that $\left(u_{T}^{\ell}, F_{T}^{e}\right), \ell=1,2$ are $(\beta, N)$-practically stable for any bounded neighborhood of the origin $N \subset \mathbb{R}$. (The results of [14] also allow us to conclude that, using either of the 


\begin{tabular}{||c|c|c|c|c||}
\hline Sampling period & Euler based & 2nd order & Damping control & Improvement of 2nd order \\
\hline $\mathrm{T}=0.01$ & {$[-19.1 ; 19.1]$} & {$[-21 ; 21]$} & {$[-8.2,8.2]$} & $9 \%$ \\
\hline $\mathrm{T}=0.1$ & {$[-5.1 ; 5.1]$} & {$[-5.8 ; 5.8]$} & {$[-2.5,2.5]$} & $14 \%$ \\
\hline $\mathrm{T}=0.5$ & {$[-1.8 ; 1.8]$} & {$[-2.3 ; 2.3]$} & {$[-1,1]$} & $22 \%$ \\
\hline $\mathrm{T}=1$ & {$[-1 ; 1]$} & {$[-1.5 ; 1.5]$} & {$[-0.55,0.55]$} & $50 \%$ \\
\hline
\end{tabular}

Table 1: Comparison of regions of attractions for Euler and 2nd order based controllers.

controllers (61), (62), we achieve semi-global-practical stability of the sampled-data model of the plant by choosing $T$ sufficiently small.)

For the purpose of comparison we considered also a continuous-time globally asymptotically stabilizing, damping control law [9, Example 13.5] for the same system implemented using a sampler and zero order hold

$$
u(k)=-x(k)-3 x^{3}(k) .
$$

It can be shown that this control law asymptotically stabilizes the approximate Euler model semi-globally. The closed-loop Euler approximate model with the controller (63) is:

$$
x(k+1)=(1-T) x(k)-2 T x^{3}(k) .
$$

In order to illustrate the performance of the controllers (61), (62) and (63) which are respectively applied to (57) (using a sampler and zero order hold), we estimated regions of attraction for several sampling periods and the results of simulations are presented in Table 1. Simulations were carried out using SIMULINK package in MATLAB with the parameters: Runge-Kutta 5, tolerance 0.001, min step size 0.00001, max step size 10 .

The 2nd order controller gives the largest regions of attraction for all tested sampling periods and the improvement over the Euler controller is presented in the last column of the table. However, the 2nd order controller is more complicated and, in general, more difficult to design than the Euler-based controller. The damping control law for the system gives almost twice smaller regions of attraction compared to the Euler-based controller for all the sampling periods in Table 1. However, all three controllers do achieve semi-global-practical stabilization of the sampled-data closed-loop system (semi-global in the sampling period).

Example 2 Consider the two-input linear system

$$
\begin{aligned}
& \dot{x}_{1}=x_{1}+u_{1} \\
& \dot{x}_{2}=u_{2}
\end{aligned}
$$

which has exact discretization

$$
\begin{aligned}
& x_{1}(k+1)=e^{T} x_{1}(k)+\left[e^{T}-1\right] u_{1}(k) \\
& x_{2}(k+1)=x_{2}(k)+T u_{2}(k)
\end{aligned}
$$

and Euler approximate discretization

$$
\begin{aligned}
& x_{1}(k+1)=[1+T] x_{1}(k)+T u_{1}(k) \\
& x_{2}(k+1)=x_{2}(k)+T u_{2}(k) .
\end{aligned}
$$

Consider the controller

$$
u(x)= \begin{cases}{\left[\begin{array}{c}
-2 x_{1} \\
0
\end{array}\right]} & \text { if } 0<0.1 x_{1}<x_{2}<10 x_{1} \\
{\left[\begin{array}{c}
-2 x_{1} \\
-x_{2}
\end{array}\right]} & \text { otherwise. }\end{cases}
$$


$\mathbf{A 1}, \overline{\mathbf{A}}$ : It follows from Lemma 1 that $\left(u_{T}, F_{T}^{a}\right)$ is one-step consistent with $\left(u_{T}, F_{T}^{e}\right)$. However, $\left(u_{T}, F_{T}^{a}\right)$ is not multi-step consistent with $\left(u_{T}, F_{T}^{e}\right)$. Indeed, consider the initial condition $\left(\xi_{1}, \xi_{2}\right)=$ $(1,0.1)$. It is easy to see that, in this case, $\left(x_{1}^{a}(k, \xi), x_{2}^{a}(k, \xi)\right)=(1-T)^{k}(1,0.1)$, i.e., the positive ray $x_{2}=0.1 x_{1}>0$ is forward invariant for all $T \in(0,1)$. On the other hand, $\left(x_{1}^{e}(1, \xi), x_{2}^{e}(1, \xi)\right)=$ $\left(\left(2-e^{T}\right) 1,(1-T) 0.1\right)$, i.e., for all small $T>0, x_{2}^{e}(1, \xi)<10 x_{1}^{e}(1, \xi)$ and $x_{2}^{e}(1, \xi)>0.1 x_{1}^{e}(1, \xi)$ since $e^{T}>1+T$. It follows that, for $k \geq 1, x(k, \xi)$ will take values on the horizontal line given by $x_{2}=(1-T) 0.1$ moving in the direction of decreasing $x_{1}$ until it crosses the positive ray $x_{2}=10 x_{1}$. Let $\bar{k}$ denote the number of steps required to cross the positive ray $x_{2}=10 x_{1}$. It is easy to put an upper and lower bound on $\bar{k} T$ that is independent of $T$. Then since, for all $k \leq \bar{k}$, we have $x_{2}^{e}(k, \xi)=(1-T) 0.1$ while $x_{2}^{a}(k, \xi)=(1-T)^{k} 0.1 \leq e^{-k T} 0.1$, it is clear that the conclusion of Lemma 2 is not satisfied. Hence $\left(u_{T}, F_{T}^{a}\right)$ cannot be multi-step consistent with $\left(u_{T}, F_{T}^{e}\right)$.

B1: We take $V_{T}(x)=\left|x_{1}\right|+\left|x_{2}\right|$. We get, for $T \in(0,1)$ :

$$
0<0.1 x_{1}<x_{2}<10 x_{1} \quad \Longrightarrow \quad V_{T}\left(F_{T}^{a}\left(x, u_{T}(x)\right)\right)-V(x)=-T\left|x_{1}\right| \leq-\frac{T}{20}\left[\left|x_{1}\right|+\left|x_{2}\right|\right]
$$

and, otherwise,

$$
V_{T}\left(F_{T}^{a}\left(x, u_{T}(x)\right)\right)-V(x)=-T\left[\left|x_{1}\right|+\left|x_{2}\right|\right] .
$$

It follows that the family $\left(u_{T}, F_{T}^{a}\right)$ is EGAS by equi-Lipschitz Lyapunov functions.

C1: We conclude from Theorem 2 (and also using the homogeneity of $V_{T}(x)$ and $F_{T}^{e}\left(x, u_{T}(x)\right.$ ) to pass from a semiglobal practical result to a global result and following the steps of the proof of Theorem 2 to get an exponential result) that the family $\left(u_{T}, F_{T}^{e}\right)$ is $\left(\beta, \mathbb{R}^{2}\right)$-stable with $\beta(s, t)$ of the form $k s \exp (-\lambda t)$ with $k>0$ and $\lambda>0$.

Example 3 (A, $\overline{\mathbf{B}}, \overline{\mathbf{C}})$ Consider the double integrator, its Euler approximation and its exact discretetime model:

$$
\text { double integrator: } \begin{aligned}
\dot{x}_{1} & =x_{2} \\
\dot{x}_{2} & =u \\
\text { approximate: } x_{1}(k+1) & =x_{1}(k)+T x_{2}(k) \\
x_{2}(k+1) & =x_{2}(k)+T u(k) \\
\text { exact: } x_{1}(k+1) & =x_{1}(k)+T x_{2}(k)+0.5 T^{2} u(k) \\
x_{2}(k+1) & =x_{2}(k)+T u(k) .
\end{aligned}
$$

The following controller is designed for the Euler model:

$$
u(x)=-\frac{x_{1}}{T}-\frac{2 x_{2}}{T} .
$$

$\overline{\mathbf{C}}$ : The eigenvalues of the exact closed-loop are $\lambda_{1}=1-\frac{T}{2}, \lambda_{2}=-1, \forall T>0$ and thus the exact closed-loop model is not $(\beta, N)$-practically stable for any pair $(\beta, N)$.

A : The eigenvalues of the Euler closed-loop system are $\lambda_{1}=+\sqrt{1-T}, \lambda_{2}=-\sqrt{1-T}$. In a similar way as in the previous example we can show that there exists $b>0$ such that for all $T \in] 0,0.5[$ we have:

$$
|x(k)| \leq b \exp (-0.5 k T)|x(0)|, \forall x(0) \in \mathbb{R}^{2} .
$$

Hence, the approximate closed-loop system is $\left(\beta, \mathbb{R}^{2}\right)$-stable with $\beta(s, t):=b \exp (-0.5 t)$.

$\overline{\mathbf{B}}$ : It now follows from Theorem 1 that $\left(u_{T}, F_{T}^{a}\right)$ is not multi-step consistent with $\left(u_{T}, F_{T}^{e}\right)$. In fact, $\left(u_{T}, F_{T}^{a}\right)$ is not one-step consistent with $\left(u_{T}, F_{T}^{e}\right)$ since

$$
\left|\epsilon_{1}(x)\right|=\left|T^{2} / 2\left(-x_{1} / T-2 x_{2} / T\right)\right|=2 T\left|x_{1}+x_{2}\right|, \forall x \in \mathbb{R}^{2}, \forall T .
$$




\section{Conclusions}

In this paper we have presented results that justify the design of nonlinear sampled-data controllers based on approximate discrete-time models for sufficiently fast sampling. We have shown that with the appropriate combinations of consistency (of the approximate discrete-time model with the exact discrete-time model) and stability uniformity (in the sampling period for the family of approximate discrete-time closed-loops), semiglobal practical asymptotic stability of the origin of the sampled-data control system is guaranteed. Like in the continuous-time case, separate tools (like small gain theorems, Lyapunov conditions or homogeneity) should be used to analyze local asymptotic stability properties (cf. $[20])$.

The results that we have presented for asymptotic stability of the origin can easily be extended to arbitrary compact attractors and/or the asymptotic stability assumption on the family of approximate discrete-time models can be relaxed to a practical asymptotic stability assumption.

\section{References}

[1] B. Castillo, S. Di Gennaro, S. Monaco and D. Normand-Cyrot, On regulation under sampling, IEEE Trans. Automat. Contr., 42 (1997), pp. 864-868.

[2] T. Chen and B. A. Francis, Optimal sampled-data control systems. Springer-Verlag: London, 1995.

[3] F. H. Clarke, Y. S. Ledyaev, E. D. Sontag and A. I. Subbotin, Asymptotic controllability implies feedback stabilization, IEEE Trans. Automat. Contr., 42 (1997), pp. 1394-1407.

[4] D. Dochain and G. Bastin, Adaptive identification and control algorithms for nonlinear bacterial growth systems, Automatica, 20 (1984), pp. 621-634.

[5] G. F. Franklin, J. D. Powell and M. L. Workman, Digital control of dynamic systems. AddisonWesley Pub. Co. Inc.: Reading, 1990.

[6] G. C. Goodwin, B. McInnis and R. S. Long, Adaptive control algorithm for waste water treatment and $p H$ neutralization, Optimal Contr. Applic. Meth., 3 (1982), pp. 443-459.

[7] L. Grüne, Asymptotic controllability and exponential stabilization of nonlinear control systems at singular points, SIAM J. Contr. Optimiz., 36 (1998), pp. 1485-1503.

[8] N. Kazantzis and C. Kravaris, System-theoretic properties of sampled-data representations of nonlinear systems obtained via Taylor-Lie series, Int. J. Control, 67 (1997), pp. 997-1020.

[9] H. K. Khalil, Nonlinear systems. Prentice-Hall: New Jersey, 1996.

[10] B. C. Kuo, Digital control systems. Saunders College Publishing: Ft. Worth, 1992.

[11] Y.Lin, E.D.Sontag and Y.Wang, A smooth converse Lyapunov theorem for robust stability, SIAM J. Contr. Opt., 34 (1996), pp. 124-160.

[12] I. M. Y. Mareels, H. B. Penfold and R. J. Evans, Controlling nonlinear time-varying systems via Euler approximations, Automatica, 28 (1992), pp. 681-696.

[13] S. Monaco and D. Normand-Cyrot, Sampling of a linear analytic control system, Proc. CDC'85, pp. 1457-1462.

[14] D. Nešić, A. R. Teel and E.D.Sontag, Formulas relating $\mathcal{K} \mathcal{L}$ stability estimates of discrete-time and sampled-data nonlinear systems, to appear in Syst. Contr. Lett., 1999.

[15] R. Ortega and D. Taoutaou, A globally stable discrete-time controller for current-fed induction motors, Systems and Control Letters, 28 (1996), pp. 123-128. 
[16] D. H. Owens, Y. Zheng and S. A. Billings, Fast sampling and stability of nonlinear sampled-data systems: Part 1. Existence theorems, IMA J. Math. Contr. Informat., 7 (1990), pp. 1-11.

[17] Z. Qu, Robust control of nonlinear uncertain systems. John Wiley \& Sons: New York, 1998.

[18] E. D. Sontag, Smooth stabilization implies coprime factorization, IEEE Trans. Automat. Contr., 34 (1989), pp. 435-443.

[19] E. Sontag. Comments on integral variants of ISS. Systems \& Control Letters, 34 (1998), 93-100.

[20] A.R. Teel and L. Praly. Tools for semiglobal stabilization by partial state and output feedback, SIAM J. Control Optim., vol. 33, no. 5, pp. 1443-1488, September 1995.

[21] A.R. Teel and L. Praly. A smooth Lyapunov function from a class- $\mathcal{K} \mathcal{L}$ estimate involving two positive semidefinite functions. Submitted.

[22] S. A. Svoronos, D. Papageorgiou and C. Tsiligiannis, Discretization of nonlinear control systems via the Carleman linearization, Chemical Eng. Science, 49 (1994), pp. 3263-3267.

[23] A. M. Stuart and A. R. Humphries, Dynamical systems and numerical analysis. Cambridge University Press: New York, 1996.

[24] A. R. Teel, D. Nešić and P. V. Kokotović, A note on input-to-state stability of sampled-data nonlinear systems, In Proc. IEEE Conf. on Dec. and Control, Tampa, Florida, 1998, pp. 2473-2478.

[25] Y. Zheng, D. H. Owens and S. A. Billings, Fast sampling and stability of nonlinear sampled-data systems: Part 2: Sampling rate estimation, IMA J. Math. Contr. Informat., 7 (1990), pp. 13-33. 